\title{
The evolution of weak Mg II absorption lines
}

\author{
Toru Misawa ${ }^{1}$, Ryan Lynch $^{1}$, Anand Narayanan ${ }^{1}$, Nikola \\ Milutinović ${ }^{1}$, Tae-Sun $\mathrm{Kim}^{2}$, and Jane C. Charlton ${ }^{1}$ \\ ${ }^{1}$ Department of Astronomy and Astrophysics, Pennsylvania State University, \\ University Park, PA 16802, USA \\ ${ }^{2}$ Institute of Astronomy, Madingley Road, Cambridge CB3 0HA, UK
}

\begin{abstract}
We present preliminary results of two surveys of weak $\mathrm{Mg}$ II (rest frame equivalent width $\left.W_{r}(2796)<0.3 \AA\right)$ absorbers at $\langle z\rangle=0.15$ and at $\langle z\rangle=1.75$. For the low redshift survey, we used $25 \mathrm{HST} / \mathrm{STIS}$ echelle quasar spectra $(R=45,000)$ that covered Si II $\lambda 1260$ and C II $\lambda 1335$ at $0<z<0.3$. These transitions were used as a tracer of $\mathrm{Mg}$ II $\lambda 2796$, allowing a survey of redshift path-length $g(z)=5.3$. Our high redshift survey covers a redshift path-length of $g(z)=6.7$ in 15 high resolution QSO spectra obtained with UVES on VLT. We find that $d N / d z=0.96 \pm 0.19$ for $0<z<0.3$ and $0.02<W_{r}(2796)<0.3 \AA$, and $d N / d z=1.05 \pm 0.16$ for $1.4<z<2.2$ over the same equivalent width range. These numbers are to be compared to the results of a previous survey of weak $\mathrm{Mg}$ II absorbers: $d N / d z=1.74 \pm 0.10$ for $0.4<z<1.4$ (Churchill et al. 1999). There appears to be a peak epoch for weak $\mathrm{Mg}$ II absorbers at $z \sim 0.9$. At higher redshifts, the observed value is significantly less than expected for cosmological evolution, and somewhat smaller than expected considering the larger extragalactic background radiation at $\langle z\rangle=1.75$. We conclude from this that the processes that produce weak $\mathrm{Mg}$ II absorbing structures are equally, or somewhat less, active at $\langle z\rangle=1.75$ than at $\langle z\rangle=0.9$. At lower redshifts, $\langle z\rangle=0.15$, we would expect a larger number of weak Mg II absorbers than observed (comparing to intermediate redshifts). A significant fraction of these expected objects would result from the evolution of low density structures in which only C IV absorption was observable at higher redshifts. The overall evolution of weak $\mathrm{Mg}$ II absorbers is consistent with them being related to some type of global star formation activity, perhaps restricted to certain environments. More detailed examination of the evolution of the properties of weak $\mathrm{Mg}$ II absorbers is needed.
\end{abstract}

\section{The nature of weak Mg II absorbers}

Weak Mg II absorbers have metallicities that approach or exceed the Solar value (Rigby, Charlton \& Churchill 2002; Charlton et al. 2003). Despite these high metallicities, weak $\mathrm{Mg}$ II absorbers are not found within $50 \mathrm{~h}^{-1} \mathrm{kpc}$ of giant luminous galaxies. They may be related to supernova activity and/or star formation activity in dwarf galaxies and/or the cosmic web. Weak Mg II absorption appears to be produced by regions of the order of 1-100 pc (Rigby et al. 2002; Charlton et al. 2003). The same absorbers also show $\mathrm{C}$ IV absorption, which is thought to be related to a larger, high ionisation region at the same velocity. Flattened geometries are favoured by the number statistics and kinematic structures of Mg II and C IV systems (Milutinović et al. 2005).

Weak Mg II systems constitute a significant class of absorbers. Their observed redshift path density at redshift one is twice that of strong $\mathrm{Mg}$ II absorbers that are associated with luminous galaxies (Churchill et al. 1999). Churchill et al. (1999) found $d N / d z=$ $1.74 \pm 0.10$ for systems with $0.02<W_{r}(2796)<0.3 \AA$ in range $0.4<z<1.4$. Here we present preliminary results on $d N / d z$ at both lower redshifts $(0<z<0.3)$ and higher redshifts $(1.4<z<2.2)$. We also compare the observed $d N / d z$ values with expected values from the evolution of the extragalactic background and from cosmological evolution. Finally, we consider possible implications for the nature of weak Mg II absorbers 
based on the relationship between their evolution and the evolution of the global star formation rate in the Universe.

\section{Results}

\section{1. $0<z<0.3$ survey}

Spectra were obtained using the E140M grating of the STIS spectrograph on the HST. The resolution of these spectra is $\mathrm{R}=45,000$, and they cover the $\mathrm{Si}$ II $\lambda 1260$ and $\mathrm{C}$ II $\lambda 1335$ transitions in the redshift range of interest. We use these transitions as tracers of the low ionisation Mg II phase. The completeness of the survey is $30 \%$ at the minimum equivalent width limit of $\mathrm{Mg}$ II, corresponding to $W_{r}(2796)>0.02 \AA$, however, it increases rapidly for larger values. The results are not significantly sensitive to the calibration ratio of Si II and $\mathrm{C}$ II to $\mathrm{Mg}$ II that we adopt. We detected 5 weak Mg II absorbers in $20 \mathrm{HST} / \mathrm{STIS}$ spectra in a redshift path of 5.3 at $0<z<0.3$ that gives $d N / d z=0.96 \pm 0.19$ at $\langle z\rangle=0.15$ (see Fig. 1).

\section{2. $1.4<z<2.2$ survey}

Spectra were obtained using UVES on VLT. The resolution of these spectra was $\mathrm{R}=45,000$ and the spectra typically provide a search region for $\mathrm{Mg}$ II from the Lyman-alpha emission line up to $\sim 10,000 \AA$. The spectra had sufficient $S / N$ so that our survey is effectively $100 \%$ complete for $W_{r}(2796)>0.02 \AA$ lines in regions of the spectrum relatively free from atmospheric absorption. Regions contaminated by atmospheric absorption were eliminated from our redshift path. We detected 25 weak Mg II absorbers in 15 VLT QSO spectra. This includes seven weak Mg II absorbers with $1.4<z<2.2$ in a redshift path of 6.7 , which gives $d N / d z=1.05 \pm 0.16$ at $\langle z\rangle=1.75$ (see Fig. 1). We also evaluate $d N / d z$ at $0.4<z<1.4$ using our spectra, to find $d N / d z=1.57 \pm 0.14$. Our binned data over this range (shown as dotted points in Fig. 1) agree with $d N / d z=1.74 \pm 0.10$, measured by Churchill et al. (1999) (shown as solid points).
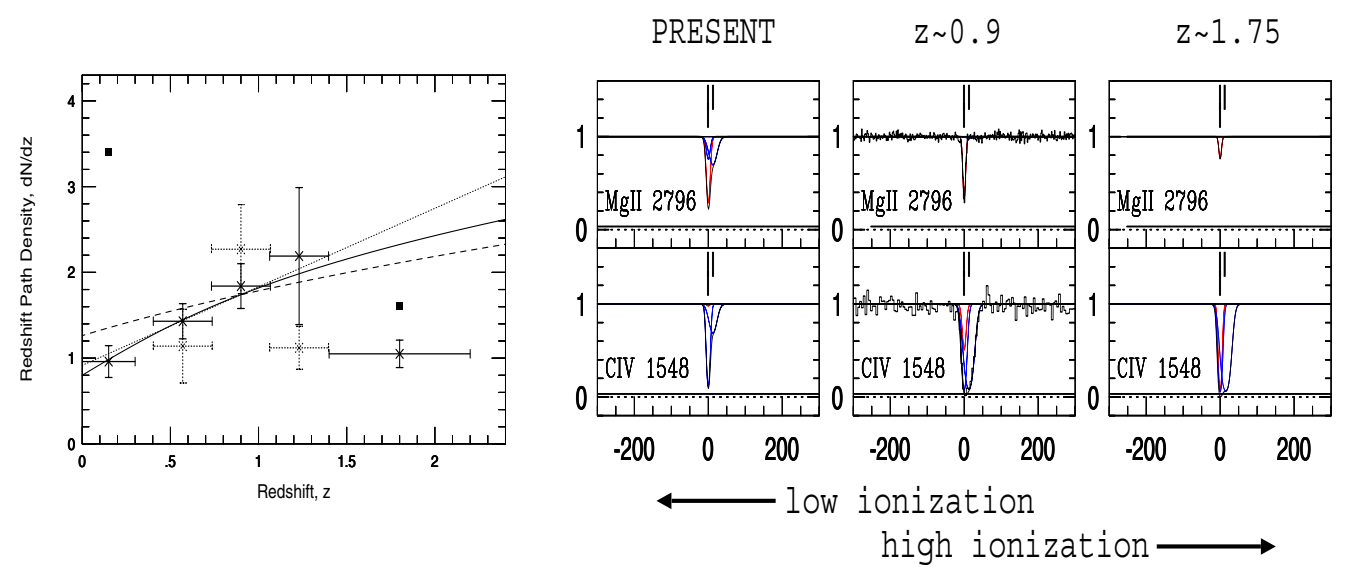

Figure 1. (left) Evolution of the weak $\mathrm{Mg}$ II absorber population, combining the results of our two surveys with the earlier survey of Churchill et al. (1999). The solid, dotted, and dashed curves represent the no-evolution expectations for a $\Lambda$ CDM Universe $\left(\Omega_{m}=0.3\right.$ and $\left.\Omega_{\Lambda}=0.7\right)$, and a critical Universe $\left(q_{0}=0\right.$ and $\left.q_{0}=0.5\right)$, respectively.

Figure 2. (right) The middle panel presents the observed profile of a weak Mg II absorber at $z=0.9$. We show both Mg II and C IV. The modelled contributions from higher and lower density regions are also overlaid. 


\section{Discussion}

The extragalactic background radiation intensity decreases by about half a dex from $\langle z\rangle=1.75$ to $\langle z\rangle=0.9$, and by almost another dex from $\langle z\rangle=0.9$ to $\langle z\rangle=0.15$ (Haardt \& Madau 2001). In the context of this evolution, we would expect that Mg II absorption is weaker at higher redshift and stronger at lower redshift for the same physical entity. To illustrate this point, we simulate the expected evolution of a real $\langle z\rangle=0.9$ weak $\mathrm{Mg}$ II system back to $z=1.75$ and forward to $z=0$, as shown in Fig. 2. We estimate the expected evolution of the weak Mg II absorber population subjected to the evolving extragalactic background radiation and cosmological evolution. We began by computing the rest frame equivalent widths of $\mathrm{Mg}$ II $\lambda 2796$ at $\langle z\rangle=0.9$ that correspond to $0.02<W_{r}(2796)<0.3 \AA$ at $\langle z\rangle=1.75$ and at $\langle z\rangle=0.15$. We then integrated the observed $\langle z\rangle=0.9$ equivalent width distribution to predict the expected number of weak $\mathrm{Mg}$ II absorbers at $\langle z\rangle=1.75$ and at $\langle z\rangle=0.15$ (solid squares in Fig. 1). The expected number at $\langle z\rangle=0.15$ also includes a dominant contribution from low density, kiloparsec-scale absorbers that produced only $\mathrm{C}$ IV absorption at $\langle z\rangle=0.9$. These predictions assume static populations of objects or ones that regenerate at constant rates.

\section{Conclusions}

Results on the evolution of weak $\mathrm{Mg}$ II lines to low redshift $(\langle z\rangle=0.15)$ are summarised as follows: (i) The observed $d N / d z$ at $\langle z\rangle=0.15$ is consistent with cosmological evolution from $\langle z\rangle=0.9$; (ii) There are fewer low- $z$ weak $\mathrm{Mg}$ II systems observed than expected, considering that some low density "C IV-only" absorbers would have observable $\mathrm{Mg}$ II due to the decreasing extragalactic background radiation. Those C IV structures are within $100 \mathrm{kpc}$ of galaxies and have been hypothesised to be related to satellites or galactic fountains (Chen, Lanzetta \& Webb 2001); (iii) The number of weak Mg II absorbers from higher density, parsec-scale structures may also be evolving/decreasing from $\langle z\rangle=0.9$ to $\langle z\rangle=0.15$; (iv) The observed evolution is consistent with a relationship between the overall star formation rate in the Universe and the generation of weak Mg II absorbers.

We also found evolutionary results for weak Mg II lines at high redshift $(\langle z\rangle=1.75)$ : (i) Processes that generate weak Mg II absorbers are similar or more probably somewhat less active at $\langle z\rangle=1.75$ than at $\langle z\rangle=0.9$; (ii) The global star formation rate in the Universe is likely to be a factor of 2 higher at $\langle z\rangle=1.75$ than at $\langle z\rangle=0.9$; (iii) Weak Mg II absorber evolution roughly traces the global star formation rate, however, small differences are not unexpected because this population is known not to be well correlated with the entire population of galaxies in which star formation is occurring.

\section{Acknowledgements}

This research was funded by NASA grants NAG 5-6399 and NNG04GE73G and by NSF grant AST-04-07138. RSL was partially funded by the NSF REU programme.

\section{References}

Charlton, J. C., Ding, J., Zonak, S. G., Churchill, C. W., Bond, N. A., Rigby, J. R., 2003, ApJ, 589,111

Chen, H.-W., Lanzetta, K. M., Webb, J. K., 2001, ApJ, 556, 158

Churchill, C. W., Rigby, J. R., Charlton, J. C., Vogt, S. S., 1999, ApJS, 120, 51

Haardt, F., Madau, P., 2001, astro-ph/0106018

Milutinović, N., Rigby, J. R., Ding, J., Masiero, J. R., Palma, C., Charlton, J. C., 2005, in preparation

Rigby, J. R., Charlton, J. C., Churchill, C. W., 2002, ApJ, 565, 743 